\title{
Frutosamina como principal parâmetro glicêmico do paciente diabético em hemodiálise
}

\author{
Fructosamine as the main glycemic marker for the diabetic patient in hemodialysis
}

\author{
Gabriel Santos Mazzaferro ${ }^{a}$, Adroaldo Lunardellib \\ a Biomédico. Centro Universitário Ritter dos Reis (UniRitter). \\ b Farmacêutico. Doutor em Saúde da Criança. Professor do Centro Universitário Ritter dos Reis (UniRitter).
}

RESUMO Introdução: A hemoglobina glicada (HbA1c) tem sido utilizada desde a metade dos anos 1970 como marcador glicêmico e é, atualmente, adotada como principal marcador glicêmico de pacientes portadores de diabetes mellitus. Porém, muitas condições tornam sua correta interpretação quase impossível e faz-se necessária a utilização de outro parâmetro, a frutosamina, exercendo o papel de principal e mais preciso marcador glicêmico de pacientes portadores de diabetes mellitus hemodialíticos.

Objetivo: O estudo objetivou revisar a literatura quanto a utilização da frutosamina como principal e mais preciso parâmetro glicêmico ao paciente hemodialítico portador da diabetes mellitus.

Materiais e Métodos: Trata-se de uma revisão integrativa da literatura onde foram acessadas as bases de dados Medline/Pubmed, Science Direct e Scielo. Utilizou-se os descritores: diabetes mellitus, frutosamina, hemoglobina glicada e hemodiálise, bem como a combinação destas palavras.

Resultados: Dos 77 artigos encontrados, foram identificados 04 estudos demonstrando a utilização da frutosamina e HbA1c em pacientes portadores de diabetes mellitus hemodialíticos. Destes estudos avaliados, apenas um apontou a HbA1c como o melhor marcador de acompanhamento glicêmico. Porém, quase metade dos pacientes avaliados neste estudo estavam recebendo dose de ferro via intravenosa para tratamento da anemia.

Conclusão: Conclui-se que a frutosamina é o marcador mais adequado e o mais preciso para o controle glicêmico de pacientes portadores de diabetes mellitus hemodialíticos em comparação à hemoglobina glicada. Dos quatro artigos selecionados, nenhum estudo foi realizado na população brasileira, mostrando a importância de estudos serem realizados a respeito da utilização da frutosamina em pacientes hemodialíticos portadores da diabetes mellitus no Brasil.

Palavras-chave: diabetes mellitus; frutosamina; hemoglobina glicada; hemodiálise.

Introduction: Glycated hemoglobin (HbA1c) has been used since mid 70's as a glycemic marker and it is, currently, adopted as the main glycemic marker to diabetes mellitus patients. However, many conditions make this accurate interpretation nearly impossible and it is required the use of another marker, fructosamine, which functions as the main and most accurate glycemic marker for hemodialytic patients with diabetes mellitus.

Objective: The present study aimed to review the literature regarding the use of fructosamine as the main and most accurate glycemic marker to the hemodialytic patient with diabetes mellitus.

Materials and Methods: It is an integrative literature review conducted by searching the databases Medline/Pubmed, Science Direct and Scielo. The following descriptors were used: diabetes mellitus, fructosamine, glycated hemoglobin and hemodialysis, as well as the combination of these words.

Results: From the 77 articles found, 04 studies were identified demonstrating the use fructosamine and HbA1C in hemodialytic patients with diabetes mellitus. From these evaluated studies, only one pointed the HbA1C as the best glycemic marker. However, almost half of the patients evaluated on that study were receiving intravenous iron doses to anemia treatment.

Conclusion: It possible to conclude that fructosamine is the most suited and the most accurate glycemic marker to hemodialytic patients with diabetes mellitus. Of the four selected articles, none of them was conducted in the Brazilian population, showing the importance of further studies about the use of fructosamine in hemodialytic patients with diabetes mellitus in Brazil.

Keywords: diabetes mellitus; fructosamine; glycated hemoglobin; hemodialysis.

\section{Correspondência:}

ADROALDO LUNARDELLI

Laboratório de Análises Clínicas do Centro Universitário Ritter dos Reis (UniRitter)

Rua Orfanotrófio, 555 - bloco C, 2 o andar

90840-440 Porto Alegre, RS, Brasil

E-mail: adroaldo_lunardelli@uniritter.edu.br 


\section{INTRODUÇÃO}

A diabetes mellitus é uma doença crônica caracterizada por altos níveis de glicose no sangue (glicemia) em jejum ${ }^{1}$ classificada em dois tipos: tipo I (um), caracterizado como diabetes mellitus insulinodependente, causada devido a incapacidade de secreção do hormônio insulina por parte do pâncreas; e tipo II (dois), diabetes mellitus não insulinodependente. Trata-se, este tipo, do mais comum, correspondendo a aproximadamente de 90-95\% do número total de casos $^{2}$ e sua origem ocorre através da combinação entre resistência à ação da insulina e da resposta secretora compensatória inadequada deste hormônio.

Pacientes portadores do diabetes mellitus tipo II evoluem, ao longo do tempo, para a diabetes mellitus tipo I. Isto se deve ao fato de que a constante resistência à insulina seja responsável por gerar a hiperinsulinemia, ou seja, no aumento da concentração desse hormônio no plasma. A falência das células beta pancreáticas no diabetes mellitus tipo II ocorre quando as mesmas se tornam incapazes de fazer a compensação da resistência à insulina ${ }^{3}$. Esta falência é progressiva com perda de células beta ${ }^{4}$, particularmente quando a hiperglicemia está estabelecida ${ }^{3}$. Em seguida, há diminuição da secreção de insulina devido à progressiva redução da função das células beta (responsáveis pela produção da insulina), havendo hipossensibilidade destas células à glicose, fato este relacionado ao esgotamento funcional ocasionado pela hiperestimulação inicial ao avançar da idade do indivíduo acometido pela doença.

É classificado como diabético todo paciente que apresenta níveis glicêmicos acima de $126 \mathrm{mg} / \mathrm{dL}$ em estado de jejum de oito horas, sendo a amostra obtida em duas dosagens em momentos diferentes (sendo visto em pacientes não portadores da doença valores abaixo de $100 \mathrm{mg} / \mathrm{dL}$ nessa condição); igual ou acima de 200 mg/dL em coleta aleatória; e superior a $200 \mathrm{mg} / \mathrm{dL}$ duas horas após a ingestão de $75 \mathrm{~g}$ de glicose (valor para pacientes saudáveis: menos de $140 \mathrm{mg} / \mathrm{dL}$ ao mesmo teste). Há, ainda, os pré-diabéticos, com valores entre 100 e $125 \mathrm{mg} / \mathrm{dL}$ em jejum de oito horas ou valores entre 140 e $200 \mathrm{mg} / \mathrm{dL}$ em testes de tolerância à glicose ${ }^{5}$.

A hemoglobina glicada, também conhecida por HbA1c ou, ainda, A1c, foi identificada, inicialmente, como uma hemoglobina "anormal" em pacientes diabéticos por Rahbar et al. ${ }^{6}$ na década de 1960. A HbA1c se refere a porção da hemoglobina que se liga à glicose presente na corrente sanguínea. A hemácia tem tempo de vida médio de 117 dias para homens e 106 dias para mulheres, ambos em indivíduos não diabéticos ${ }^{7} \mathrm{e}$, durante este período, vai incorporando glicose para realização de suas atividades.
Desta maneira, é possível avaliar o nível médio da glicemia dos últimos dois a três meses do paciente, pois altas taxas glicêmicas durante este período resultarão em altos níveis nos valores da hemoglobina glicada ${ }^{8}$. Por este motivo esse marcador é largamente utilizado na prática clínica como acompanhamento dos níveis de glicemia do paciente diabético ${ }^{9}$. Apesar de hemácias em condições normais apresentarem esse tempo médio de vida, a hemoglobina glicada é melhor acompanhada laboratorialmente quando quantificada mensalmente, pois o valor médio da glicose do último mês contribui para, aproximadamente, metade dos valores de $\mathrm{HbA}_{1} \mathrm{c}^{10}$. Os $50 \%$ restantes estão divididos entre os dias $31-90$, correspondendo a $40 \%$, e os últimos $10 \%$ entre os 91-120 dias ${ }^{11}$. Em uma amostra de sangue haverão eritrócitos em diferentes tempos de vida, sendo a maior prevalência hemácias mais jovens e com diferentes níveis de exposição à hiperglicemia ${ }^{12}$.

A frutosamina é formada através do mecanismo não enzimático que envolve a ligação da glicose sérica às proteínas séricas, em especial a albumina, para formar cetaminas ${ }^{13}$. Em outras palavras, é o produto da glicação de proteínas séricas. Possui tempo de vida médio entre 14 e 21 dias, ou seja, duas a três semanas, e reflete o nível médio de glicemia deste período ${ }^{14}$. A frutosamina eleva-se na presença de níveis aumentados de glicose no sangue ${ }^{15}$, indicando que o controle glicêmico foi inadequado ${ }^{16}$ no período supracitado. Sua utilização ocorre, principalmente, nas circunstâncias em que o uso da HbA1c não é válida devido interferentes, como pacientes que apresentam qualquer fator que seja responsável pela alteração no tempo de vida das hemácias como, por exemplo: anemia, talassemias, diminuição dos valores de eritropoetina, nefropatia diabética, diabetes mellitus gestacional ${ }^{17}$, e/ou indivíduos em tratamento por hemodiálise ${ }^{18}$. Formação de tipos diferentes de hemoglobina também se torna um interfente na correta interpretação dos valores de $\mathrm{HbA}_{1} \mathrm{c}^{12}$. Em outras palavras, qualquer fator que seja responsável pela alteração do tempo de vida das hemácias ou que resulte em sua alteração estrutural, torna-se um interferente na interpretação dos valores de HbA1c.

No acompanhamento de pacientes com nefropatia diabética e/ou com evolução ao tratamento por hemodiálise, diversos interferentes são responsáveis por ocasionar na errônea interpretação da HbA1c, atualmente o principal analito utilizado no controle dos níveis glicêmicos de indivíduos diabéticos. Diante disso, o presente estudo objetivou revisar a literatura quanto a utilização da frutosamina como principal e mais preciso parâmetro de acompanhamento dos níveis glicêmicos ao paciente diabético em hemodiálise. 


\section{MATERIAIS E MÉTODOS}

O presente estudo consiste em uma revisão integrativa da literatura com base na busca de artigos científicos disponíveis em diferentes bancos de dados. A busca foi realizada entre os meses de agosto a novembro de 2015 nas bases da dados Medline/Pubmed e Science Direct, nos idiomas português e inglês, abrangendo artigos publicados entre 2002 a 2015. Utilizou-se os descritores: diabetes mellitus, frutosamina, hemoglobina glicada e hemodiálise e a combinação destas palavras, em inglês (diabetes mellitus, fructosamine, glycated hemoglobin e hemodialysis).

Foi adotado como critério de inclusão as publicações cuja temática abordou os resultados da utilização de marcadores laboratoriais de acompanhamento diabético em pacientes submetidos à hemodiálise em protocolos clínicos humanos. Primeiramente, foram analisados títulos e resumos dos resultados das buscas, sendo selecionados aqueles estudos que contemplaram a temática proposta, incluídos e lidos de forma detalhada. Foram excluídos da seleção estudos realizados em pacientes não diabéticos e com animais. A análise seguiu referencial teórico, incluindo a leitura exploratória, seletiva, analítica e interpretativa do material obtido.

\section{RESULTADOS E DISCUSSÃO}

Através das buscas realizadas e de acordo com os critérios descritos anteriormente, foram encontrados 77 trabalhos (53 artigos na base de dados Science Direct e 24 na base de dados Medline/Pubmed). Deste total, 29 estudos foram excluídos por restrição de data, restando 48 artigos potencialmente elegíveis. Dentre estes, quatro trabalhos contemplaram os critérios de inclusão do presente estudo, e são ressaltados na Tabela 1. Os estudos compararam os principais parâmetros glicêmicos de avaliação a fim de ressaltar qual o mais indicado ao acompanhamento de pacientes portadores de diabetes mellitus hemodialíticos. Notadamente, há pouca literatura disponível acerca desta temática, o que reflete no pequeno número de publicações encontradas dedicada a este tema.
Até novembro de 2014, segundo a World Health Organization (WHO), haviam cerca de 247 milhões de diabéticos no mundo, onde $80 \%$ do número de óbitos está diretamente ligado a complicações ocasionadas pela própria doença e encontra-se, principalmente, em países de renda baixa e média - atual estado econômico do Brasil. Estudos da organização mostram que a diabetes mellitus estará, em 2030, entre as sete principais doenças a causarem óbito no mundo ${ }^{19}$. No que diz respeito aos países das Américas, os casos de diabetes mellitus foram estimados em 35 milhões no ano de 2000 e em 64 milhões para 2025. Isso resultará no aumento de $82 \%$ em apenas 25 anos. Segundo King et al. ${ }^{20}$, nos países desenvolvidos, será observado aumento no número de casos em adultos, enquanto nos países em fase de desenvolvimento, espera-se observar o fenômeno em todas as faixas etárias, sendo a principal entre os 45 a 64 anos de idade, onde a prevalência deverá triplicar, e entre 20 a 44 anos, junto com indivíduos acima de 65 anos, onde espera-se que este valor duplique.

A doença renal tem início em indivíduos diabéticos devido ao descontrole crônico dos níveis glicêmicos, acarretando em lesões. Essas lesões não atingem apenas os rins, como também são frequentemente vistas no sistema cardíaco, em especial coração e artéria aorta; na artéria renal, gerando aterosclerose da artéria renal; olhos, principalmente retina, cristalino e íris; e incidência de lesões no sistema nervoso entre $40 \%$ a $60 \%$ em pacientes diabéticos ${ }^{4}$. Além da diabetes mellitus, outras condições como hipertensão arterial, glomerulonefrite e doença renal policística ${ }^{21}$ apresentaram relativa correlação à doença renal crônica (DRC), sendo denominados como fatores agravantes para o aparecimento desta. Vale ressaltar que a diabetes mellitus é a principal doença causadora da DRC 22 .

Cada rim possui entre 1 e 1,5 milhão de néfrons unidade funcional filtradora dos rins - onde cada uma dessas estruturas é independente. Temos como funções destas unidades funcionais: capacidade de depuração seletiva dos resíduos presentes no sangue, manter a água essencial, além de realizar o equilíbrio eletrolítico do organismo. Em outras palavras, os rins mantém o equilíbrio homeostático do corpo.

Tabela 1. Trabalhos selecionados e contemplados no presente estudo com respectivos autores, títulos, ano e períodico de publicação.

\begin{tabular}{|c|c|c|}
\hline Autores & Título e Ano & Periódico \\
\hline Mittman et al. ${ }^{43}$ & $\begin{array}{l}\text { Serum fructosamine versus glycosylated hemoglobin as an index of glycemic control, } \\
\text { hospitalization, and infection in diabetic hemodialysis patients (2010) }\end{array}$ & Kidney Int \\
\hline Joy et al. ${ }^{47}$ & Long-term glycemic control measurements in diabetic patients receiving hemodialysis (2002) & Am J Kidney Dis \\
\hline Mácsai et al. ${ }^{48}$ & Significance of hemoglobin A1c in the management of diabetes in dialysis patient (2014) & Orv Hetil \\
\hline Williams et al. ${ }^{49}$ & $\begin{array}{l}\text { The glycemic indices in dialysis evaluation (GIDE) study: Comparative measures of glycemic } \\
\text { control in diabetic dialysis patients (2015) }\end{array}$ & Hemodialysis International \\
\hline
\end{tabular}


Tudo isso através das funções de fluxo sanguíneo renal, filtração glomerular, reabsorção tubular e, por fim, secreção tubular. Quando há perda de grande parte funcional renal, restando apenas cerca de $10 \%$, os rins perdem a capacidade de filtração eficaz do sangue, sendo então necessária a utilização de outros métodos, como a hemodiálise (HD) para a manutenção da vida deste paciente. Nesta técnica, uma máquina é responsável por bombear o sangue através de um capilar até o dialisador, com função de retirar toxinas presentes no sangue, sendo levado, após esta etapa, para uma câmara que detecta e rompe partículas de ar antes que o sangue seja levado ao organismo do paciente novamente. A maioria dos pacientes diabéticos quando desenvolve doença renal terminal ingressa em programas de hemodiálise ${ }^{23}$.

A nefropatia diabética é a maior complicação microvascular da diabetes mellitus ${ }^{24}$, sendo uma condição que resulta na alteração dos vasos sanguíneos renais, levando à perda de proteína na urina. Nessa ordem, quando não controlada, pode levar o órgão à redução de sua função lenta e progressivamente. A nefropatia diabética é diagnosticada entre $20 \%$ a $40 \%$ dos pacientes com diabetes mellitus, tanto do tipo I quanto do tipo II ${ }^{25}$. Já em pacientes diabéticos em estágio final da doença renal - ou seja, em hemodiálise este número sobre para $30 \%$ a $50 \%$, o que representa a prevalência de mais de um em cada quatro pacientes ${ }^{26}$. Esta condição, além da elevada prevalência, é associada à alta frequência no número de óbitos causados por outras doenças, sendo as doenças cardíacas as mais observadas ${ }^{27}$.

Em pacientes portadores de nefropatia diabética, a utilização da $\mathrm{HbA1}$ c não é adequada, pois o tempo de vida e o turnover das hemácias estão alterados. Os elevados índices de produtos urêmicos causam a diminuição do tempo de meia-vida das hemácias, além disso, a lesão nos rins afeta a sua produção de eritropoietina, hormônio responsável por estimular a produção de novos eritrócitos. Esses fatores levam a uma queda do percentual de hemoglobina circulante na corrente sanguínea ${ }^{27}$. Além disso há resposta inflamatória por consequência da lesão renal, onde há aumento da produção de hepcidina, um peptídeo produzido no fígado que inibe a absorção duodenal de ferro, além de inibir a mobilização de ferro de seus estoques, resultando, por sua vez, em anemia ${ }^{29}$, caracterizada como normocrômica e normocítica ${ }^{30}$, na nefropatia diabética causada, principalmente, pela diminuição da produção de eritropoetina, constatando um grande número de interferentes para a utilização da HbA1c, sendo observadas interpretações errôneas do controle glicêmico. Em casos como este é recomendado o acompanhamento desses pacientes através da dosagem de outro marcador, a frutosamina, refletindo as variações glicêmicas das últimas duas semanas do indivíduo.
Estudo realizado por Draczeviski et al. ${ }^{30}$ constatou que a anemia está presente na maioria dos pacientes portadores da DRC, sendo esta uma das manifestações mais comuns entre os indivíduos acometidos por esta condição. Sua causa pode ocorrer de duas maneiras: pela diminuição da produção de eritropoetina pelos rins, sendo esta a principal causa da disfunção renal; e/ou, ainda, diminuição das concentrações de ferro ${ }^{29}$. Estudos demonstraram que há importante associação entre o estágio da doença renal com a intensidade e prevalência da anemia ${ }^{31}$. A redução do número de néfrons potencializa o processo de fibrose túbulo-intersticial das nefropatias crônicas através da elevação do stress oxidativo e hipóxia, causados pela diminuição do potencial antioxidante do sangue ocasionado, por sua vez, pela anemia, responsável por gerar o aumento de espécies reativas de oxigênio ${ }^{32}$, caracterizando em uma síntese diminuida de eritropoetina nos rins ${ }^{33}$. Em outras palavras, pacientes com DRC anêmicos possuem perda da função renal mais rapidamente e necessitam iniciar o programa de diálise anteriormente, se comparados a pacientes com DRC não anêmicos ${ }^{29,34}$.

Uma série de interferentes podem ser observados no que diz respeito à correta utilização da $\mathrm{HbA} 1 \mathrm{c}$, como a diminuição dos valores de eritropoetina, devido a deficiência de vitamina B12 e/ou ferro, e/ou anemia aplásica, levando ao aumento no número de eritrócitos antigos circulantes e, desta forma, em um progressivo aumento dos valores de $\mathrm{HbA} \mathrm{c}^{35}$. Isto se deve ao fato de que a hemoglobina, em eritrócitos mais antigos, é mais glicosilada em relação às hemoglobinas dos reticulócitos - eritrócitos imaturos - mais jovens ${ }^{36}$. Portanto, se o tempo-médio de vida das células vermelhas for curta, como no caso da anemia hemolítica ou em uma recuperação recente de uma perda aguda de sangue, então os valores de HbA1c serão falsamente menores, independentemente da glicemia ${ }^{37}$. Porém, se a idade-média da população de células vemelhas circulantes for maior, então haverá elevação dos níveis de HbA1c, também de forma independente da glicemia.

Fatores como hemoglobinopatias (anemia falciforme e talassemias), também tornam dificultosa a interpretação dos valores da hemoglobina glicada devido alteração estrutural das hemácias ${ }^{37}$. Alterações químicas e fatores genéticos de hemoglobina recombinantes também podem influenciar os resultados da $\mathrm{HbA} 1 \mathrm{c}$, como é o que ocorre em fatores étnicos, por exemplo. Além da glicação normal da HbA0 (fração não glicada da hemoglobina) em HbA1c, há a glicação de outros produtos derivados, como é o que ocorre, por exemplo, na formação da: $\mathrm{HbC}$ em populações de descendência africana; $\mathrm{HbD}$, ocorrida em populações indianas; HbE, observada comumente em populações asiáticas; e, ainda, HbS, célula falsiforme, que são formadas em adição à $\mathrm{HbA}^{3} \mathrm{c}^{38}$. Posto 
que nosso país, Brasil, possui uma grande miscigenação, estas ocasiões podem ser casualmente encontradas. Tanto a $\mathrm{HbC}$ quanto a $\mathrm{HbD}$ resultam em HbA1c falsamente diminuida, enquanto a $\mathrm{HbE}$ não altera os parâmetros de HbA1c. A HbS, por sua vez, é responsável por acarretar em valores falsamente aumentados desse parâmetro ${ }^{39}$. Níveis elevados de hemoglobina fetal, HbF, utilizada através do medicamento hidroxiureia para tratamento da anemia falciforme, por exemplo, pode resultar na diminuição dos

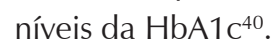

Ingestão crônica de aspirina e/ou altas doses de vitamina E e C, assim como outros antioxidantes, podem diminuir a concentração de HbA1c pois causam a inibição de glicação da hemoglobina ${ }^{41}$. Porém, permance incerto qual destes fenômenos é o responsável por gerar a diferente valorização da HbA1c na prática clínica ${ }^{12}$. Observou-se que elevados níveis de bilirrubina também ocasionam o aumento artificial dos valores da $\mathrm{HbA}_{1} \mathrm{c}^{35}$. Com a crescente diminuição da função renal há o desenvolvimento da acidose sistêmica e isto contribui ao falso aumento dos valores da $\mathrm{HbA}^{4} \mathrm{c}^{42}$.

Estudo realizado por Mittman et al. ${ }^{43}$ mostrou que a utilização da frutosamina refletiu melhor que a HbA1c nos níveis glicêmicos de pacientes diabéticos em tratamento por hemodiálise. É conveniente ressaltar que os autores indicam que a frutosamina deve ser corrigida de acordo com a concentração plasmática de albumina, pois este procedimento a torna mais fidedigna com o perfil de acompanhamento do paciente diabético. Isto se deve ao fato de que em pacientes diabéticos dialíticos, tanto em hemodiálise como em diálise peritonial, a leitura da HbA1c pode ser falsamente baixa, sendo sua acurácia questionada para estes casos, em especial em pacientes com função renal tão baixa ${ }^{18}$. Esse resultado ocorre devido ao fato de que os pacientes portadores de
DRC apresentam, além da redução da eritropoetina, níveis aumentados de hemoglobina carbamilada (hemoglobina modificada quimicamente presente, principalmente, em pacientes urêmicos, resultando da ligação entre ureia e $\mathrm{Hb})^{44}$ e exposição variável à glicose exógena através da solução dialítica ${ }^{45} \mathrm{e}$ devido à acidose sistêmica ${ }^{46}$. Os interferentes responsáveis por alterar os valores da HbA1c e o que estes ocasionam são relatados na Tabela 2.

Joy et al. ${ }^{47}$ verificaram, em estudo realizado em 2002, que valores da $\mathrm{HbA} 1 \mathrm{c}$ entre $6,0 \%$ a $7,0 \%$ (onde valores entre $6,5 \%$ a $7,0 \%$ representam bom controle dos níveis de $\mathrm{HbA} 1 \mathrm{c}$ em se tratando de pacientes portadores de diabetes mellitus) estimaram os valores glicêmicos de forma similar a pacientes sem DRC. Porém, em valores acima de 7,5\% resultaram em valores superestimados de $\mathrm{HbA} 1 \mathrm{c}$ em pacientes portadores de DRC. O estudo foi realizado em apenas 23 pacientes diabéticos em tratamento por hemodiálise em comparação a um grupo controle composto por oito pacientes não portadores de diabetes mellitus dialíticos. Em outro estudo, Mácsai et al. ${ }^{48}$ aponta que a margem de HbA1c associada ao baixo risco de mortalidade encontra-se entre 6,5\% e 8,0\% para pacientes com diálise peritoneal. O trabalho mostra a frutosamina como uma grande alternativa, mas aponta fragilidades nos dois marcadores.

Outro estudo realizado por Williams et al. ${ }^{49}$, por outro lado, mostrou que a HbA1c foi o parâmetro que melhor representou os níveis glicêmicos dos pacientes avaliados em ambos os grupos estudados - pacientes em hemodiálise e diálise peritoneal. Ou seja, a HbA1c foi o analito que teve melhor correlação com os valores médios de glicose em relação à frutosamina e albumina glicada. Porém, deve-se avaliar que quase metade dos 1758 pacientes estudados número exato não fornecido pelo pesquisador - estavam

Tabela 2. Principais variantes interferentes na correta determinação da hemoglobina glicada.

\begin{tabular}{|c|c|c|c|}
\hline Autor & Ano & Variante & $\begin{array}{l}\text { Principais achados nos valores da } \\
\text { hemoglobina glicada (HbA1c) }\end{array}$ \\
\hline Gallagher et al. ${ }^{35}$ & 2009 & Diminuição de eritropoetina & Falsamente alta \\
\hline Gallagher et al. ${ }^{35}$ & 2009 & Deficiência de ferro e/ou vitamina B12 & Falsamente alta \\
\hline Gallagher et al..$^{35}$ & 2009 & Anemias e talassemia & Falsamente alta \\
\hline Unnikrishnan et al. ${ }^{40}$ & 2012 & Hemoglobina Fetal (HbF) & Falsamente baixa \\
\hline Schnedl et al. ${ }^{39}$ & 2001 & Hemoglobina C ( $\mathrm{HbC})$ & Falsamente baixa \\
\hline Schnedl et al. ${ }^{39}$ & 2001 & Hemoglobina S (HbS) & Falsamente alta \\
\hline Schnedl et al. ${ }^{39}$ & 2001 & Hemoglobina D (HbD) & Falsamente baixa \\
\hline Inaba et al. ${ }^{18}$ & 2007 & Pacientes hemodialíticos & Falsamente baixa \\
\hline Gallagher et al. ${ }^{35}$ & 2009 & Elevação dos valores de bilirrubina & Falsamente alta \\
\hline Ghigo et al. ${ }^{42}$ & 2014 & Acidose sistêmica & Falsamente alta \\
\hline $\operatorname{Radin}^{17}$ & 2014 & Diabetes gestacional & Falsamente baixa \\
\hline Radin $^{17}$ & 2014 & Elevadas doses de aspirina, vitaminas $\mathrm{C}$ e/ou $\mathrm{E}$, antioxidantes & Falsamente baixa \\
\hline
\end{tabular}


recebendo doses de ferro via intravenosa para manutenção da anemia, sendo este um interferente no real valor da HbA1c, uma vez que a administração de ferro irá refletir na diminuição dos valores deste analito. Outro importante fator que se deve destacar é que não foram incluídos no estudo pacientes com doença renal crônica, outro fator que fez com que os valores da hemoglobina glicada refletissem melhor os níveis glicêmicos em relação à frutosamina.

Diferentemente da HbA1c, a frutosamina não possui tantos fatores que possam comprometer sua utilização. Quando há quaisquer interferentes que alterem os valores de HbA1c, este parâmetro deve ser inutilizado, sendo a frutosamina o mais aconselhado e preciso analito de acompanhamento dos níveis glicêmicos. Os níveis anormais de frutosamina maternos podem ser considerados marcadores de anormalidades cardíacas fetais ${ }^{16}$. Em se tratando de pacientes hemodialíticos portadores de diabetes mellitus, deve-se levar em consideração que estes indivíduos possuem anemia e deficiência de eritropoetina, dois interferentes que impossibilitam a correta interpretação dos valores de HbA1c. Além disso, vale ressaltar que a mensuração da frutosamina é rápida, de baixo custo quando comparada a outros métodos com a mesma finalidade e altamente específica, não havendo interferentes como o urato e triglicerídeos, por exemplo ${ }^{50}$, além de permitir a avaliação do resultado mais rapidamente a respeito dos métodos terapêuticos empregados ${ }^{51}$.

Concluindo, a frutosamina é um parâmetro normalmente complementar, mas que se torna fundamental e utilizado como principal e mais adequado marcador dos níveis glicêmicos nas diversas ocasiões onde o uso da HbA1c não é válido devido a seus diversos interferentes como, por exemplo, anemia e deficiência de eritropoetina, ambas situações presentes em pacientes portadores de diabetes mellitus em tratamento por hemodiálise. Esse analito não apenas é mais preciso que a HbA1c, como também é capaz de detectar o resultado das manobras terapêuticas mais precocemente que a hemoglobina glicada permitindo, ainda, a avaliação e melhor manejo a respeito do tratamento desses pacientes.

\section{REFERÊNCIAS}

1. Otunctemur A, Ozbek E, Sahin S, Dursun M, Besiroglu H, Koklu I, Erkoc M, Danis E, Bozkurt M, Gurbuz A. Diabetes mellitus as a risk factor for high grade renal cell carcinoma. Asian Pac J Cancer Prev. 2014;15(9):3993-6. http://dx.doi.org/10.7314/ APJCP.2014.15.9.3993

2. Winkelmann ER, Fontela PC. Condições de saúde de pacientes com diabetes mellitus tipo 2 cadastrados na Estratégia Saúde da Família, em ljuí, Rio Grande do Sul, 2010-2013. Epidemiol Serv Saúde. 2014;23(4):665-74. http://dx.doi.org/10.5123/S167949742014000400008
3. Prentki $\mathrm{M}$, Nolan $\mathrm{CJ}$. Islet b cell failure in type 2 diabetes. J Clin Invest. 2006 July;116(7):1802-12. http://dx.doi.org/10.1172/ $\mathrm{JCl} 29103$

4. Nolan CJ, Augusto VD. Reversibility of defects in proinsulin processing and islet b-cell failure in obesity-related type 2 diabetes. Diabetes. 2016;65(2):352-4. http://dx.doi.org/10.2337/dbi15-0020

5. Juarez DT, Demaris KM, Goo R, Mnatzaganian CL, Smith HW. Significance of $\mathrm{HbA1C}$ and its measurement in the diagnosis of diabetes mellitus: US experience. Diabetes Metab Syndr Obes. 2014;7:487-94. http://dx.doi.org/10.2147/DMSO.S39092

6. Rahbar S, Blumenfeld O, Ranney HM. Studies of an unusual hemoglobin in patients with diabetes mellitus. Biochem Biophys Res Commun. 1969;36(5):838-43. http://dx.doi.org/10.1016/0006291X(69)90685-8

7. Jeffcoate SL. Diabetes Control and Complications: the role of glycated heamoglobin, 25 years on. Diabet Med. 2004;21(7): 657-65. http://dx.doi.org/10.1046/j.1464-5491.2003.01065.x

8. Camargo JL, Gross JL. Glico-hemoglobina (HbA1c): Aspectos Clínicos e Analíticos. Arq Bras Endocrinol Metab. 2004;48:451-63. http://dx.doi.org/10.1590/S0004-27302004000400005

9. American Diabetes Association (ADA); Tests of glycemia in diabete: position statement. Diabetes Care. 2003;26:S106-8. http://dx.doi. org/10.2337/diacare.26.2007.S106

10. Calisti L, Tognetti S. Measure of glycosylated hemoglobin. Acta Biomed. 2005;76(3):59-62.

11. Henrichs HR. HbA1c-glycated Hemoglobin and Diabetes Mellitus. Bremen: UNI-MED; 2009.

12. Speeckaert M, Biesen WV, Delanghe J, Slingerland R, Wiecek A, Heaf J, Drechsler C, Lacatus R, Vanholder R, Nistor I. Are there better alternatives than heamoglobin A1c to estimate glycaemic control in the chronic kidney disease population? Nephrol Dial Transplant. 2014;29(12):2167-77. http://dx.doi.org/10.1093/ndt/ gu006

13. Armbruster DA. Fructosamine: structure, analysis, and clinical usefulness. Clin Chem. 1987;33(12):2153-63.

14. Nansseu JRN, Fokom-Domgue J, Noubiap JJN, Balti EV, Sobngwi E, Kengne AP. Fructosamine measurement for diabetes mellitus diagnosis and monitoring: a systematic review and metaanalysis protocol. BMJ Open. 2015;5(5):e007689. http://dx.doi. org/10.1136/bmjopen-2015-007689

15. Nagasaka Y, Fujii S, Yaga K, Matsumura S, Kaneko T. Clinical application of measuring serum fructosamine as an index of glycemic control in diabetic patients. Bull Yamaguchi Med. Sch. 1988; 35(3-4):59-62.

16. Reis ZSN, Miranda APB, Rezende CAL, Detofol RB, Costa CR, Cabral ACV. Rastreamento de cardiopatias congênitas associadas ao diabetes mellitus por meio da concentração plasmática materna de frutosamina. Rev Bras Ginecol Obstet. 2010;32(2):66-71. http:// dx.doi.org/10.1590/S0100-72032010000200003

17. Radin MS. Pitfalls in hemoglobin A1c measurement: When results may be misleading. J Gen Intern Med. 2014;29(2):388-94. http:// dx.doi.org/10.1007/s11606-013-2595-x

18. Inaba $M$, Okuno $S$, Kumeda $Y$, Yamada $S$, Imanishi $Y$, Tabata $T$, Okamura M, Okada S, Yamakawa T, Ishimura E, Nishizawa Y. 
Glycated albumin is a better glycemic indicator than glycated hemoglobin values in hemodialysis patients with diabetes: effect or anemia and erythropoietin injection. J Am Soc Nephrol. 2007;18(3):896-903. http://dx.doi.org/10.1681/ASN.2006070772

19. World Health Organization. [Internet]. 2014. [acesso em 2014 nov. 18]; Disponível em: http://www.who.int/mediacentre/factsheets/ fs $312 /$ en/

20. King $\mathrm{H}$, Aubert RE, Herman WH. Global burden of diabetes, 1995-2025. Diabetes Care. 1998;21(9):1414-31. http://dx.doi. org/10.2337/diacare.21.9.1414

21. Ribeiro RCHM, Santiago E, Bertolin DC, Ribeiro DF, Cesarino CB, Burdmann EA. Depressão em idosos portadores de insuficiência renal crônica em tratamento hemodialítico. Acta Paul Enferm. 2009;22:505-8. http://dx.doi.org/10.1590/S010321002009000800010

22. Mehdi U, Toto RD. Anemia, diabetes, and chronic kidney disease. Diabetes Care. 2009;32(7):1320-6. http://dx.doi.org/10.2337/ dc08-0779

23. Angelo BZ, Tessarollo C, Carvalho VD, Lemos TC, Koch MC, Maraschin JF. Fatores preditores de mortalidade em pacientes diabéticos em terapia de substituição renal em Tubarão -SC. ACM Arq Catarin Med. 2010;39(4):23-8.

24. Bouvet BR, Paparella CV, Arriaga SMM, Monje AL, Amarilla AM, Almará AM. Evaluation of urinary $\mathrm{N}$-acetyl-betaD-glucosaminidase as a marker of early renal damage in patients with type 2 diabetes mellitus. Arq Bras Endocrinol Metab. 2014;58(8):798-801. http:// dx.doi.org/10.1590/0004-2730000003010

25. Collins AJ, Foley RN, Herzog C, Chavers B, Gilbertson D, Ishani A, Kasiske B, Liu J, Mau LW, McBean M, Murray A, St Peter W, Guo H, Gustafson S, Li Q, Li S, Li S, Peng Y, Qiu Y, Roberts T, Skeans M, Snyder J, Solid C, Wang C, Weinhandl E, Zaun D, Arko C, Chen SC, Dalleska F, Daniels F, Dunning S, Ebben J, Frazier E, Hanzlik C, Johnson R, Sheets D, Wang X, Forrest B, Constantini E, Everson S, Eggers P, Agodoa L. US Renal Data System 2010 Annual Data Report. Am J Kidney Dis. 2011;57(1 Suppl 1):A8, e1-526. http:// dx.doi.org/10.1053/j.ajkd.2010.10.007

26. American Diabetes Association. Standards of medical care in diabetes - 2012. Diabetes Care. 2012 Jan;35 Suppl 1:S11-63. http://dx.doi.org/10.2337/dc12-s011

27. Tzamaloukas AH, Friedman EA. Diabetes In: Daugirdas JT, Blake PG, Ing TS. Manual de diálise. 3를 ed. Rio de Janeiro: Medsi; 2003.

28. National Kidney Foundation. Anemia and Chronic Kidney Disease. 33th ed. New York: National Kidney Foundation; 2006-2007.

29. Abensur H. Deficiência de ferro na doença renal crônica. Rev Bras Hematol Hemoter. 2010;32(2):84-8. http://dx.doi.org/10.1590/ s1516-84842010005000047

30. Draczevski L, Teixeira LM. Avaliação do perfil bioquímico e parâmetros hematológicos em pacientes submetidos à hemodiálise. Rev Saúde Pesq. 2011;4(1):15-22.

31. Bueno CR, Frizzo MN. Anemia na doença renal crônica em hospital da região noroeste do estado do Rio Grande do Sul. J Bras Nefrol. 2014;36(3):304-14.

32. Fronza BS, Winkelmann ER, Schneider J, Frizzo MN. Avaliação laboratorial em pacientes com doença renal crônica submetidos à hemodiálise. Rev Bras Anal Clin. 2014;46(1-4):30-6.
33. Miranda SP, Macedo RN, Júnior GBS, Daher EF. Síndrome síndrome cardiorrenal cardiorrenal: fisiopatologia e tratamento. Rev Assoc Med Bras. 2009;55(1):89-94. http://dx.doi.org/10.1590/S010442302009000100022

34. Gouva C, Nikolopoulos P, Ionnidis JP, Siamopoulos KC. Treating anemia early in renal failure patients slows the decline of renal function: a randomized controlled trial. Kidney Int. 2004;66(2):75360. http://dx.doi.org/10.1111/j.1523-1755.2004.00797.x

35. Gallagher EJ, Le Roith D, Bloomgarden Z. Review of hemoglobin A1c in the management of diabetes. J Diabetes. 2009;1(1):9-17. http://dx.doi.org/10.1111/j.1753-0407.2009.00009.x

36. Saudek CD, Brick JC. The clinical use of hemoglobin A1C. J Diabetes Sci Technol. 2009;3(4):629-34. http://dx.doi. org/10.1177/193229680900300402

37. Saudek CD, Derr RL, Kalyani RR. Assessing glycemia in diabetes using self-monitoring blood glucose and hemoglobin A1c. JAMA. 2006;295(14):1688-97. http://dx.doi.org/10.1001/ jama.295.14.1688

38. Lynn B, Chen PC, Sacks DB. Effects of hemoglobin variants and chemically modified derivatives on assays for glycohemoglobin. Clin Chem. 2001;47(2):153-63.

39. Schnedl WJ, Liebminger AL, Roller RE, Lipp RW, Krejs GJ. Hemoglobin variants and determination of glycated hemoglobin (HbA1c). Diabetes Metab Res Rev. 2001;17(2):94-8. http://dx.doi. org/10.1002/dmrr.186

40. Unnikrishnan R, Anjana RM, Mohan V. Drugs affecting HbA1c levels. Indian J Endocrinol Metab. 2012;16(4):528-31. http://dx.doi. org/10.4103/2230-8210.98004

41. Netto AP, Andriolo A, Filho FF, Tambascia M, Gomes MB, Melo M, Sumita NM, Lyra R, Cavalcanti S. Atualização sobre hemoglobina glicada (HbA1C) para avaliação do controle glicêmico e para o diagnóstico do diabetes: aspectos clínicos e laboratoriais. J Bras Patol Med Lab. 2009;45(1):31-48. http://dx.doi.org/10.1590/S167624442009000100007

42. Ghigo E, Porta M. Diabetes secondary to endocrine and pancreatic disorders, 22a ed. Switzerland: Karger; 2014.

43. Mittman N, Desiraju B, Fazil I, Kapupara H, Chattopadhyay J, Jani CM, Avram MM. Serum fructosamine versus glycosylated hemoglobin as an index of glycemic control, hospitalization, and infection in diabetic hemodialysis patients. Kidney Int Suppl. 2010;(117):S41-5. http://dx.doi.org/10.1038/ki.2010.193

44. Weykamp, CW, Miedema K, De Haan T, Doelman CJA. Carbamylated hemoglobin interference in glycohemoglobin assays. Clin Chem. 1999;45(3):438-40.

45. Selvaraj N, Bobby Z, Sathiyapriya V. Effect of lipid peroxides and antioxidants on glycation of hemoglobin: an in vitro study on human erythrocytes. Clin Chim Acta. 2006;366(1-2):190-5. http://dx.doi. org/10.1016/j.cca.2005.10.002

46. Sharif A, Baboolal K. Diagnostic application of the A1C assay in renal disease. J Am Soc Nephrol. 2010;21(3):383-5. http://dx.doi. org/10.1681/ASN.2010010031

47. Joy MS, Cefalu WT, Hogan SL, Nachman PH. Long-term glycemic control measurements in diabetic patients receiving hemodialysis. Am J Kidney Dis. 2002;39(2):297-307. http://dx.doi.org/10.1053/ ajkd.2002.30549 
48. Mácsai E, Rakk E, Milénder M, Fulcz A. Significance of hemoglobin A1C in the management of diabetes in dialysis patients. Orv Hetil. 2014;155(36):1421-5. http://dx.doi.org/10.1556/OH.2014. 29986

49. Williams ME, Mittman N, Lin MA, Brennan JI, Mooney A, Johnson $\mathrm{CD}$, Jani CM, Maddux FW, Lacson E. The glycemic indices in dialysis evaluation (GIDE) study: Comparative measures of glycemic control in diabetic dialysis patients. Hemodial Int. 2015;19(4):562-71. http://dx.doi.org/10.1111/hdi.12312
50. Baker JR, O'Connor JP, Metcalf PA, Lawson MR, Johnson $R N$. Clinical usefulness of estimation of serum fructosamine concentration as a screening test for diabetes mellitus $\mathrm{Br}$ Med J (Clin Res Ed). 1983;287(6396):863-7. http://dx.doi.org/10.1136/ bmj.287.6396.863

51. Kang DS, Park J, Kim JK, Yu J. Clinical usefulness of the measurement of serum fructosamine in childhood diabetes mellitus. Ann Pediatr Endocrinol Metab. 2015;20(1):21-6. http://dx.doi.org/10.6065/ apem.2015.20.1.21 